\title{
Optical Study of Liquid Crystal Lens Doped with Multiwalled Carbon Nanotubes
}

\author{
Hui LI ${ }^{1,2,4}{ }^{*}$, Fan PAN ${ }^{3}$, Yuntao WU ${ }^{1,2}$, Yanduo ZHANG ${ }^{1,2}$, Xiaolin XIE $^{4}$ \\ ${ }^{1}$ School of Computer Science and Engineering, Wuhan Institute of Technology, Wuhan 40073, P. R. China \\ ${ }^{2}$ Hubei Key Laboratory of Intelligent Robot, Wuhan 40073, P. R. China \\ ${ }^{3}$ College of Post and Telecommunication, Wuhan Institute of Technology, Wuhan 40073, P. R. China \\ ${ }^{4}$ School of Chemistry and Chemical Engineering, Huazhong University of Science and Technology, Wuhan 40073, P. R. \\ China \\ cross $^{\text {ref }}$ http://dx.doi.org/10.5755/j01.ms.22.2.12911
}

Received 20 August 2015; accepted 21 October 2015

\begin{abstract}
In this paper, a new kind of electrically controlled liquid crystal lens, which respond in a relatively fast time, is presented. The multiwalled carbon nanotubes are doped into liquid crystal to fabricate the liquid crystal lens. As $0.02 \%$ concentration of multiwalled carbon nanotubes is uniformly distributed in the liquid crystal, the optical features of the liquid crystal lens are obviously improved. The liquid crystal lens with a diameter of $2.0 \mathrm{~mm}$ was fabricated with about $0.2 \mathrm{~s}$ response time and less than $5 \mathrm{Vrms}$ applied voltage. The focal length can vary from 16 to $510 \mathrm{~mm}$, and the operation voltage changes from 1.0 to $5.5 \mathrm{Vrms}$. This liquid crystal lens has the very attractive feature of submillisecond response time, which is a much faster response time in comparison with conventional liquid crystal lens. Thus, this kind of liquid crystal lens has high potential for implementation in many practical imaging applications and imaging commercialisation.

Keywords: liquid crystal material, doped multi-walled carbon nanotubes, fast response, lens design, tuneable focal length.
\end{abstract}

\section{INTRODUCTION}

Different kinds of lenses are widely used in applications like communication, imaging systems, and so on [1]. However, conventional lenses are made of glass, which have a fixed focal length. The system for tuneable focal length is normally big, heavy, and complicated, which is not useful for modern optical devices. In the $21 \mathrm{st}$ century, compact, light, and simple optical systems are urgently needed. Material of Liquid crystal (LC) has been applied to optical device in decades. Based on LC, the LC lens has many advantages, such as tuneable focusing, voltage actuation, low power consumption, simple fabrication, compact structure, and good stability [2-4]. However, there are still some bottlenecks of the LC lens: for example, a slow response time [5]. This is a hotresearch topic for LC lens. Usually, the improved structures are intended to solve this problem, which leads to a complicated structure followed by a complicated flow of fabrication [6]. Then, the LC doped with nanoparticiples seems to be another optional method to solve that problem [7].

In this paper, we propose a relatively easy means to solve this problem. To the best of our knowledge, the LC lens doped with carbon nanotubes (CNT) is first proposed. LC doped with multiwalled carbon nanotubes (MWCNT) is utilised to replace pure LC material. A CNT is a tubeshaped material made of carbon that has a diameter measuring on the nanometer scale [7]. As the dopant MWCNT could hinder the migration of ions in LC, the features of the mixtures will be changed. There are still

\footnotetext{
* Corresponding author. Tel.: +86-27-87992007; fax: +86-27-87992007 E-mail address: lihui00317@163.com (H. Li)
}

some technological problems in doping MWCNT with LC, especially agglomeration $[8,9]$. Alcohol is utilised to lower agglomeration, which could help MWCNT to disperse well in LC. Thus, utilising alcohol could solve agglomeration. There is also the other approach to improve the electro-optical features, adopting polymer to form polymer-dispersed LC (PDLC)/ polymer -network LC (PNLC) $[10,11]$. But, the proposed method in this work is relatively simple and effective. Doped with MWCNT, the dielectric constant of the mixture increases because of the metallic feature of MWCNT, which is the major factor improving the response time of the LC lens $[12,13]$. This LC lens has the very attractive feature of submillisecond response time, which is a relatively fast response time in comparison with conventional LC lenses of pure LC. The purpose of this work is to represent the optical features of the proposed LC lens. And the principle about improved performances is also preliminarily discussed and analyzed.

In sections 2 and 3, the fabrication and related theories are used to demonstrate that doped MWCNT influences optical performance. The optical measurements are addressed in section 4. Conclusions are discussed in the last section.

\section{EXPERIMENTAL METHODS}

In the proposed LC lens, the cross profile is depicted in Fig. 1. This lens is mainly composed of two ITO substrates and a LC layer doped with MWCNT. This doped material is MWCNT rather than single-walled carbon nanotubes (SWCNT), because MWCNT improve the performance of this lens compared to SWCNT, having a smaller surface area in the LC molecule, less surface energy, and much better dispersion in LC layer. In this 
case, the reunion phenomenon, the biggest problem in doped material, would not easily form in the LC layer, which could reduce blocking during the LC moleculerotation, resulting in a faster response time [3, 14].

The fabrication lens technological flow of the proposed LC lens is presented as follows. UVphotolithography and wet hydrochloric acid (HCL) etching on the top ITO film were used to fabricate only one roundhole electrode pattern whose diameter is $2.0 \mathrm{~mm}$. Coated both internal surfaces of those two substrates and rubbed along the $x$ direction, consequently a thin film of polyimide (PI), which forms an alignment layer, was made. As shown in Fig. $1 \mathrm{a}$, this allowed all the LC directors to produce about a $2^{\circ}$ pre-tilt angle. After the above procedures, the two substrates with the expected electrode pattern and formed alignment films composed the empty LC cell by sandwiching glass micro-spheres as the spacer. The most important fabrication step was to uniformly dope a trace of MWCNT into the LC materials with as little reunion as possible. In this paper, MWCNT was chosen from Shenzhen Nanotech Port Co. Ltd, which has features such as > $90 \mathrm{wt} . \%$, a length of $1-2 \mu \mathrm{m}$, and an outside diameter of $10-20 \mathrm{~nm}$. An appropriate amount of MWCNT was pulled into a small amount of alcohol and was continually shocked by an ultrasonic shaker for $3.5 \mathrm{~h}$. The specific amounts that obtained the best results will be discussed in detail later. Then, the processed mixtures were mixed with some commercial available LC, E7 (Merck), in a clean glass-tube. A test tube shaker further mixed the LC and MWCNT mixtures for $30 \mathrm{~min}$ at $1000 \mathrm{rmp} / \mathrm{m}$. To volatilize the alcohol, the whole materials were set at around $100{ }^{\circ} \mathrm{C}$ in an oven for $30 \mathrm{~min}$. The above steps needed to be repeated three more times to maximize the evaporation of the remaining alcohol. During these repeats, the shock time using the test tube shaker was set to $1.5 \mathrm{~h}$ for a good dispersion, as shown in Fig. $1 \mathrm{~b}$. After the relatively uniform LC materials doped with WMCNT were achieved, the material controlled at 80 degree was injected to the empty cell by capillarity. The LC cell was slowly cooled to room temperature and then sealed by AB glues. According to other critical technological parameters depicted for the LC lens, $100 \mu \mathrm{m}$ diameter microspheres of spacers was used to fabricate an empty LC cell, and those specific values of operation voltage will be given in the following section.

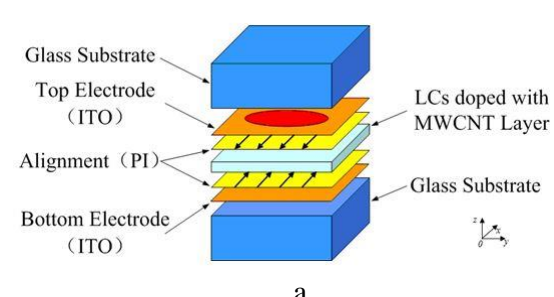

a

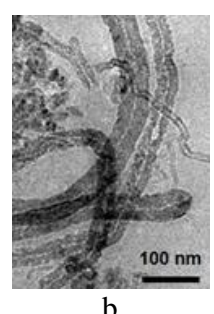

Fig. 1. Scheme of the LC lens: a-device structure of the proposed LC lens; $b$ - the scanning electron microscope image of the LC doped with MWCNT

\section{RELATED THEORY ABOUT LC LENS}

Nematic LC material has birefringence properties, which are an extraordinary refractive index and an ordinary one. Thus, as light was shone into a LC cell, this light breaks up into two rays, the ordinary one and the extraordinary one. Because this two rays travel at varied velocities, a phase delay in the LC cell will occur. When the external operation voltage is loaded, the LC molecule begins to rotate. In this way, the LC director changes too, and the degrees of the LC's tilt angle has to be considered, for the reason that an effective refractive index depends on it. For the LC material, the refractive index of the LC could be got by the following formula,

$n_{e f f}^{2}(\theta)=\frac{n_{o}^{2} n_{e}^{2}}{n_{e}^{2} \cos ^{2} \theta+n_{o}^{2} \sin ^{2} \theta}$,

where $n_{e}$ and $n_{o}$ respectively represent the extraordinary and ordinary refractive indexes. $\theta$ represents the included angle between the optical axis and the incident light.

With accordance to Section 2, a LC lens doped with a MWCNT sample can be fabricated. As the LC is the majority in the mixture by weight, the birefringence feature primarily depends on the LC. Therefore, the effect of doped MWCNT does not need to be considered. Thus, in the following analysis of the conformation of the refractive index in the LC lens, only the LC is considered. While the voltage is not applied, all LC molecules have a uniform refractive index $n_{e}$ because they are parallel to two substrates. But as soon as the operation voltage is loaded, bordered LC molecules are rotated around $90^{\circ}$. And in the central area, this refractive index remains $n_{e}$ from the start, while in the marginal area the index varies to $n_{o}$. Then, $n_{\text {eff }}(\theta)$ is the index of the transition area between the central and the marginal area. As a result, in the LC layer, a gradient distribution of the refractive index appears. As it is established, this speed of transmitted light is influenced by the refractive index. So, the LC cell will present the optical feature of the conventional convex lens, which has convergence capability for incident light.

Based on both elastic continuum and geometrical optics theory, the effective focal length of the LC lens can be calculated by the following formula [15]:

$$
f=\frac{r^{2}}{2 \Delta n \times d_{L C}},
$$

where $f$ has a relationship with the radius $(r)$, the refractive index difference $\Delta n$ (the difference between the center and margin of the aperture) and the thickness of the LC $\left(d_{L C}\right)$.

\section{RESULTS AND DISCUSSION}

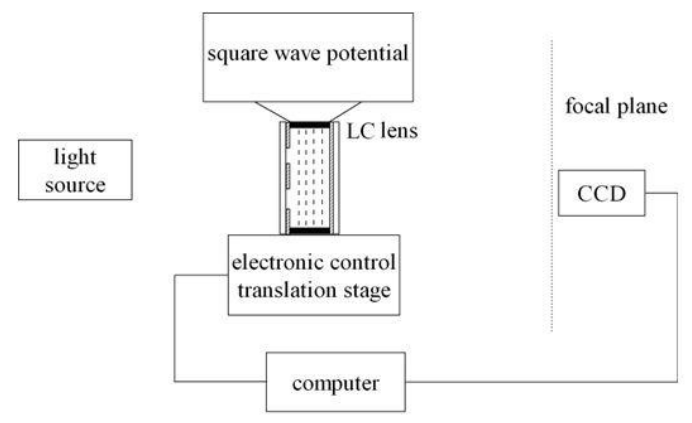

Fig. 2. Experimental setup for characterising the optical properties of the proposed LC lens 
For a purpose of presenting the optical characteristics of the LC lens, a number of experiments are given in detail. The distinguished optical characters of the LC lens doped with MWCNT are presented in this section.

Fig. 2 presents the experimental setup for characterising the optical features of the fabricated LC lens. The incident parallel white light source is symmetrical and collimated. A charge coupled device (CCD), connected to a computer, is placed at a distance of about $19.5 \mathrm{~mm}$ from the LC lens to obtain the optical focusing images.

Firstly, in order to test the classical feature of the LC lens, a light source was applied, as presented in Fig. 2. A beam profiling camera, UCD 12, was utilized to capture the focal length of the LC lens at various loaded voltages for the reason that the white light will induce a chromatic aberration. As shown in Fig. 3 with more than six tests at each voltage point, the connection between the operation voltage and the focal length was got. For the LC lens, the focal length is changed by varying the external operation voltage. And the corresponding values of the LC lens are 16 to $510 \mathrm{~mm}$ (focal length) and 1.0 to $5.5 \mathrm{Vrms}$ (operation voltage). The results show that the focal length is inversely proportional to the operation voltage. A conventional LC lens, as a comparative device, is typically fabricated by two ITO-film substrates sandwiching a nematic LC layer of $100 \mu \mathrm{m}$, and has a circular-hole electrode of $2.0 \mathrm{~mm}$ diameter and internal electrode to focus that light in order to generate effect of convex lens $[2,4,6]$. However, the threshold voltage of the LC lens doped with MWCNT is $1.0 \mathrm{Vrms}$, which is lower than $1.2 \mathrm{Vrms}$ of the conventional one as expectation. The applied voltage of the LC lens can be close to $5 \mathrm{Vrms}$, which is also better than approximately $20 \mathrm{Vrms}$ of the conventional lens. This is because the proposed LC has a mixture of the LC materials and MWCNT. The dielectric anisotropy of the mixture decreases because of the doping with MWCNT, which reduces the threshold voltage based on the equation

$V_{t h}=\pi \sqrt{\frac{4 K_{11}-2 K_{22}+K_{33}}{4 \varepsilon_{0} \Delta \varepsilon}}$,

where $K_{i i}$ represents different kinds of elastic constants, and $\varepsilon$ is the dielectric constant. According to Eq. 2, the focal length has an inverse proportion relationship between the focal length and the applied voltage, which has been proven in Fig. 3. Under the conditions of $\Delta n=0.225$ (the pure E7, $K_{11}$ is $11.1 \mathrm{pN}, K_{22}$ is $5.9 \mathrm{pN}, K_{33}$ is $17.1 \mathrm{pN}, \gamma_{1}$ is $233 \mathrm{mPa}, \varepsilon_{\|}$is $19.28, \varepsilon_{\perp}$ is $5.21, n_{e}$ is 1.7371 , and $n_{o}$ is 1.5183), $d=100 \mu \mathrm{m}, r=1 \mathrm{~mm}$, and $5.5 \mathrm{Vrms}$, for the conventional lens its focal length is about $19.6 \mathrm{~mm}$. That measured focal length value really equals the computed value, which could prove that the acquired curve could present the optical features of the LC lens. Because the lens is doped with MWCNT, the focal length range expands to $16-510 \mathrm{~mm}$, which is much wider than the conventional lens $(20-480 \mathrm{~mm})[2,4,6]$. In conclusion, MWCNT doping is proved to effectively reduce the applied voltage of the LC lens, expand the focal length range, and have no effect on the electric controlled features of the LC lens.
Secondly, response time is a very important factor for a tunable LC lens. The chosen concentration of MWCNT is about $0.02 \%$ for doping in a LC cell. In this sample, the LC lens doped with MWCNT is fabricated by the above relatively perfect doping concentration.

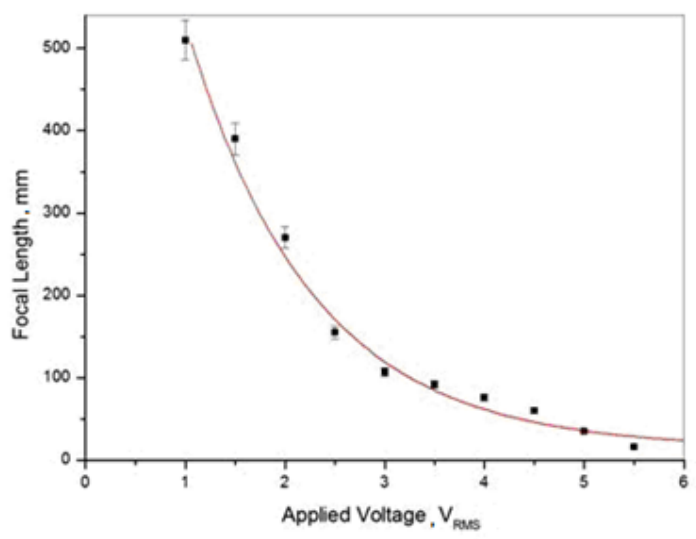

Fig. 3. The curve of the applied voltage vs. the focal length

In doer to test the response time of the LC lens, the recorded instrument, EOT-01, was used. Following the steps of the system, a halogen lamp was used as a light source for characterizing the optical transmission properties. A function generator provided an $\mathrm{AC}$ electric field (square wave, frequency of $1 \mathrm{kHz}$, voltage of $0-$ 10 Vrms) across the cell thickness. Compared the fabricated lenses with conventional ones pointed out in Table 1, the result shows that the focusing time of the proposed LC lens is significantly improved and this LC lens has only a relatively low applied voltage as expectation. In a word, for the fabricated LC lens, the focusing time is dramatically improved about $99 \%$ of and the operation voltage is reduced about $80 \%$. The structure of the two kinds of LC lenses is the same. Thus, the greatly enhanced performance of the LC lens is affected directly by the doped material. As known, MWCNT naturally presents significant metallic features. After doping it into the LC-host, the dielectric anisotropy of the mixture quickly upgrades because of its metallic features. The applied voltage of the LC lens is dramatically reduced as its dielectric anisotropy increases. On the other hand, MWCNT also has much bigger aspect ratio and greater stiffness, which will effectively facilitate the LC molecules around the MWCNT to easily align along the direction of the tube axis and opportunely contribute the rotation of the LC molecules. In addition, a polarization electric field internal LC is formed because of its metallic features. MWCNT can effectively impede the migration of ions in the LC, which can reduce the shielding effect. Therefore, the dielectric constant of the LC increases under the influence of MWCNT. As a result of the above factors, the rotation speed of the LC molecule is clearly accelerated compared to the pure LC state. In this case, the applied voltage can be reduced and the response time of the LC lens can be immediately improved under the same conditions. Therefore, the method using a trace amount of MWCNT doping can extraordinarily improve the performance of the LC lens, which can be very useful in imaging systems. 
Thirdly, a picture of a helicopter as the object is utilized to evaluate the imaging feature of the proposed LC lens.

Table 1. A comparison of focusing time and applied voltage of the conventional LC lens and proposed LC lens

\begin{tabular}{|c|c|c|c|}
\hline Compared Data & $\begin{array}{c}\text { Thickness of } \\
\text { LC cell, } \mu \mathrm{m}\end{array}$ & $\begin{array}{c}\text { Applied } \\
\text { voltage, } \\
\text { Vrms }\end{array}$ & $\begin{array}{c}\text { Focusing } \\
\text { time, } s\end{array}$ \\
\hline $\begin{array}{c}\text { Conventional LC lens } \\
\text { mentioned above }\end{array}$ & 100 & $\sim 20$ & $\sim 30$ \\
\hline Our proposed LC lens & 100 & $<5.5$ & 0.12 \\
\hline
\end{tabular}

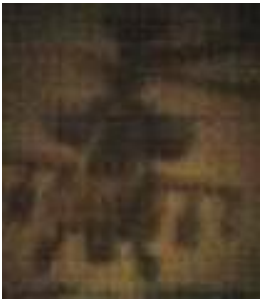

a

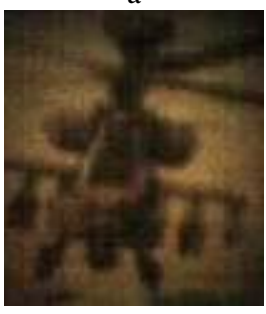

$\mathrm{c}$

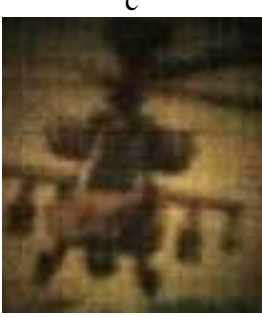

e

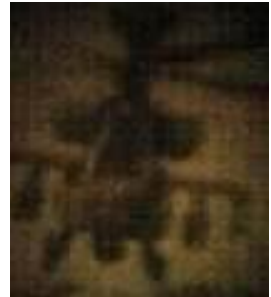

b

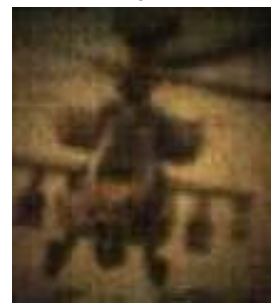

d

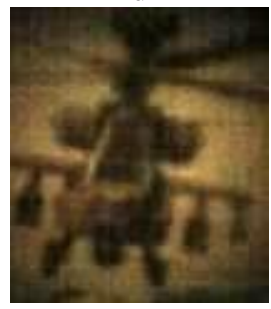

f
Fig. 4. The imaging results under different applied voltages: $\mathrm{a}-0$ Vrms; $\mathrm{b}-0.5 \mathrm{Vrms} ; \mathrm{c}-1.5 \mathrm{Vrms} ; \mathrm{d}-2.5 \mathrm{Vrms}$; e-4.0 Vrms; f-5.5 Vrms

The experiment setup without polarizations in Fig. 2 is still used. During the entire measurement process, the positions of the CCD and the LC lens are fixed without any changes. Some imaging results of the LC lens under different operation voltages are shown in Fig. 4. In those images, the acquired images under different applied voltages are all different, which means that the LC lens could be driven by the loaded voltages. At $5.5 \mathrm{Vrms}$, the image is the most clear, because in this situation the distance between the LC lens and the CCD is at the focal length under 5.5 Vrms. This could prove that the LC lens is an electrically controlled device with a feature of tunable focal length. However, these images have a relatively obvious problem: image quality of all acquired images is common. As is established, the images acquired by the conventional LC lens are as good as the images acquired by the conventional glass optical lenses. However, the proposed LC lens has a problem with image quality. In this paper, doped MWCNT is utilized to solve the technological problem of response time. The proposed LC lens is fabricated by many different factors, such as fabrication flow, cost, response time, image quality, and so on. If the above factors are considered, the proposed LC lens in this paper is just a compromise solution, with has a relatively fast response time and a common image quality. Further study is still required to study and improve its image quality in future.

Finally, the two MTF curves of the conventional LC lens and the proposed LC lens are pointed out in Fig. 5. The condition of the measurement was at $1 \mathrm{KHz}$ and 5.5 Vrms. In the figure, it is obvious that the proposed LC lens has a poorer imaging quality than the conventional LC lens $[2,4,6]$. However, the image quality of the proposed LC lens is still in an acceptable range. The reason for the poorer image quality is that MWCNT is doped into the LC lens. On one hand, MWCNT doping could improve electro-optical features effectively. On the other hand, it will lead to some disadvantages, such as optical scattering. This scattering is the major factor causing poor image quality. However, MWCNT doping overall improves the features of LC lenses in a relatively economic way.

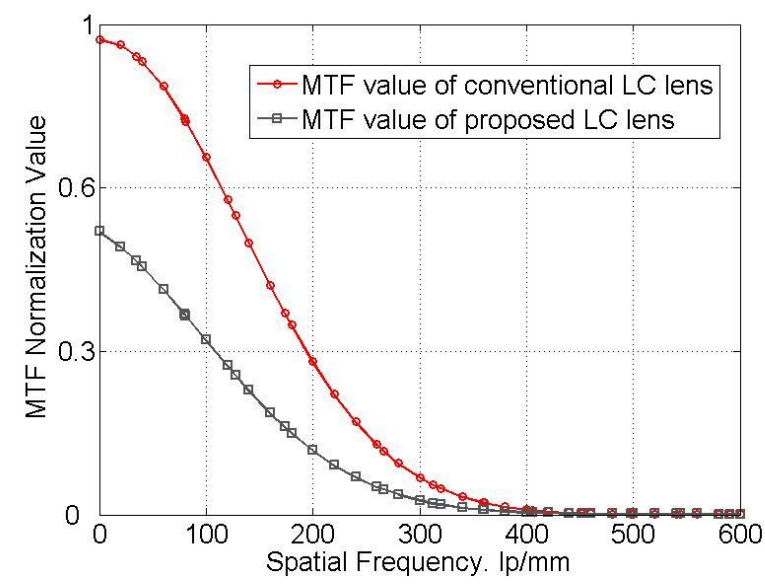

Fig. 5. The top curve shows the MTF value of the conventional LC lens, and the lower curve shows the MTF value of the proposed LC lens. Aperture size is $2.0 \mathrm{~mm}$, and CCD resolution is $50 \mathrm{lp} / \mathrm{mm}$

\section{CONCLUSIONS}

Some measurements of LC lenses, such as the relationship between operation voltage and focal length, response time, and imaging features, are obtained to study their optical performance. The experimental data represent that the LC lens doped with MWCNT has a far much faster response time than the conventional LC lens of pure LC material. After doping MWCNT into the LC-host, the dielectric anisotropy of the mixture quickly upgrades because of its metallic features. The applied voltage of the LC lens is dramatically reduced as its dielectric anisotropy increases. Based on experiments, this LC lens has the very attractive feature of a submillisecond response time, which is a relatively fast response time in comparison with the pure LC lens. In this way, this new kind of LC lens has potential for many practical imaging applications and for imaging commercialization.

\section{Acknowledgments}

This work was supported by a grant from the Research Foundation of Education Bureau of Hubei Province China (Q20141507), a grant from the Natural Science Foundation 
of Hubei Province of China (2014CFB771), China Postdoctoral Science Foundation (2014M562017), the Science Foundation of the Wuhan Institute of Technology (X2014012, and K201545), and the Open Foundation for Hubei Key Laboratory of Intelligent Robot (HBIR 201405)

\section{REFERENCES}

1. Casey, S. Liquid Lens: Advances in Adaptive Optics in Asia Communications and Photonics Conference and Exhibition Technical Digest CD FE (3) 2009: pp. 483-484.

2. Ren, H., Xu, S., Lin, Y., Wu, S.T. Adaptive Focus Lenses Optics \& Photonics News 19 (10) 2008: pp. 42-47. http://dx.doi.org/10.1364/OPN.19.10.000042

3. Mauldin, T.C., Kessler, M.R. Self-Healing Polymers and Composites International Materials Reviews 55 (6) 2010: pp. 317-346.

4. Ren, H., Wu, S.T. Introduction to Adaptive Lenses. Wiley: Hoboken, New Jersey, 2012: pp. 25-79. http://dx.doi.org/10.1002/9781118270080

5. Fowler, C.W., Pateras, E.S. Liquid Crystal Lens Review Ophthalmic \& Physioogicall Opics $10(2)$ 1990: pp. 186-194.

6. Xu, S., Li, Y., Liu, Y., Sun, J., Ren, H., Wu, S.T. FastResponse Liquid Crystal Microlens Micromachines 5 (2) 2014: pp. 300-324.

7. Baughman, R.H., Zakhidov, A.A., Heer, de W.A. Carbon Nanotubes- the Route Toward Applications Science 297 (5582) 2002: pp. $787-792$.

8. Lu, S., Chien, L. Carbon Nanotube Doped Liquid Crystal OCB Cells: Physical and Electro-Optical Properties Optics Express 16 (17) 2008: pp. 12777-12785.
http://dx.doi.org/10.1364/OE.16.012777

9. Rajasekharan, R., Dai, Q., Wilkinson, T. Electro-Optic Characteristics of a Transparent Nanophotonic Device Based on Carbon Nanotubes and Liquid Crystals Applied Optics 49 (11) 2010: pp. 2099-2104.

10. Ji, H.S., Kim, J.H., Kumar, S. Electrically Controllable Microlens Array Fabricated by Anisotropic Phase Separation from Liquid-Crystal and Polymer Composite Materials Optics Letter 28 (13) 2003: pp. 1147-1149.

11. Done, J.W. PDLC Shutters: Where has This Technology Gone Liquid Crystals $33(11-12)$ 2006: pp. 1313-1322.

12. Prakash, J., Choudhary, A., Mehta, D.S., Biradar, A.M. Effect of Carbon Nanotubes on Response Time of Ferroelectric Liquid Crystals Physical Review E 80 (1) 2009: pp. 012701-1-012701-4.

13. Leea, K.J., Parkb, H.G., Jeongb, H.C., Kimb, D.H., Seob, D.S., Leec, J.W., Moona, B.M. Enhanced ElectroOptical Behaviour of a Liquid Crystal System via Multiwalled Carbon Nanotube Doping Liquid Crystals 41 (1) 2014: pp. $25-29$. http://dx.doi.org/10.1080/02678292.2013.829248

14. Zheng, L., Li, B., Lin, P., Zhang, X., Zhang, C., Zhao, B., Wang, T. Sedimentation and Precipitation of Nanoparticles in Power-Law Fluids Microfluidics \& Nanofluidics 15 (1) 2013: pp. $11-18$. http://dx.doi.org/10.1007/s10404-012-1117-1

15. Li, H., Liu, K., Zhang, X., Zhang, T., Shen, X. Optical Focusing Feature of a Single Element in $128 \times 128$ Elements Electrically Controllable Cylindrical Liquid Crystal Lens Array Chinese Optics Letter 8 (3) 2010: pp. 329-331. http://dx.doi.org/10.3788/COL20100803.0329 